\title{
Several Differentiation Formulas of Special Functions. Part V
}

\author{
Peng Wang \\ Qingdao University of Science \\ and Technology \\ China
}

\author{
Bo Li \\ Qingdao University of Science \\ and Technology \\ China
}

Summary. In this article, we give several differentiation formulas of special and composite functions including trigonometric, polynomial and logarithmic functions.

MML identifier: FDIFF_9, version: 7.8.05 4.84.971

The articles [13], [15], [1], [16], [2], [4], [10], [11], [17], [5], [14], [12], [3], [7], [6], [9], and [8] provide the notation and terminology for this paper.

The partial function sec from $\mathbb{R}$ to $\mathbb{R}$ is defined as follows:

(Def. 1) $\sec =\frac{1}{\text { the function } \cos }$.

The partial function cosec from $\mathbb{R}$ to $\mathbb{R}$ is defined by:

(Def. 2) $\operatorname{cosec}=\frac{1}{\text { the function } \sin }$.

For simplicity, we follow the rules: $x, a, b, c$ are real numbers, $n$ is a natural number, $Z$ is an open subset of $\mathbb{R}$, and $f, f_{1}, f_{2}$ are partial functions from $\mathbb{R}$ to $\mathbb{R}$.

One can prove the following propositions:

(1) If (the function $\cos )(x) \neq 0$, then sec is differentiable in $x$ and $(\sec )^{\prime}(x)=$ $\frac{(\text { the function } \sin )(x)}{\text { (the function } \cos )(x)^{2}}$.

(2) If (the function $\sin )(x) \neq 0$, then cosec is differentiable in $x$ and $(\operatorname{cosec})^{\prime}(x)=-\frac{(\text { the function } \cos )(x)}{(\text { the function } \sin )(x)^{2}}$.

(3) $\left(\frac{1}{x}\right)_{\mathbb{Z}}^{n}=\frac{1}{x_{\mathbb{Z}}^{n}}$.

(4) Suppose $Z \subseteq$ dom sec. Then sec is differentiable on $Z$ and for every $x$ such that $x \in Z$ holds $(\sec )^{\prime}{ }_{\lceil Z}(x)=\frac{\text { (the function } \sin )(x)}{(\text { the function } \cos )(x)^{2}}$. 
(5) Suppose $Z \subseteq$ dom cosec . Then cosec is differentiable on $Z$ and for every $x$ such that $x \in Z$ holds $(\operatorname{cosec})^{\prime}{ }_{\mid Z}(x)=-\frac{(\text { the function } \cos )(x)}{(\text { the function } \sin )(x)^{2}}$.

(6) Suppose $Z \subseteq \operatorname{dom}(\sec \cdot f)$ and for every $x$ such that $x \in Z$ holds $f(x)=$ $a \cdot x+b$. Then

(i) $\sec \cdot f$ is differentiable on $Z$, and

(ii) for every $x$ such that $x \in Z$ holds $(\sec \cdot f)^{\prime}{ }_{Z Z}(x)=\frac{a \cdot(\text { the function } \sin )(a \cdot x+b)}{(\text { the function } \cos )(a \cdot x+b)^{2}}$.

(7) Suppose $Z \subseteq \operatorname{dom}(\operatorname{cosec} \cdot f)$ and for every $x$ such that $x \in Z$ holds $f(x)=a \cdot x+b$. Then

(i) $\operatorname{cosec} \cdot f$ is differentiable on $Z$, and

(ii) for every $x$ such that $x \in Z$ holds $(\operatorname{cosec} \cdot f)_{{ }^{\prime}}(x)=$ $-\frac{a \cdot(\text { the function } \cos )(a \cdot x+b)}{\text { (the function } \sin )(a \cdot x+b)^{2}}$.

(8) Suppose $Z \subseteq \operatorname{dom}\left(\sec \cdot \frac{1}{f}\right)$ and for every $x$ such that $x \in Z$ holds $f(x)=$ $x$. Then

(i) $\sec \cdot \frac{1}{f}$ is differentiable on $Z$, and

(ii) for every $x$ such that $x \in Z$ holds $\left(\sec \cdot \frac{1}{f}\right)_{{ }^{\prime}}^{\prime}(x)=$ $-\frac{(\text { the function } \sin )\left(\frac{1}{x}\right)}{x^{2} \cdot(\text { the function } \cos )\left(\frac{1}{x}\right)^{2}}$.

(9) Suppose $Z \subseteq \operatorname{dom}\left(\operatorname{cosec} \cdot \frac{1}{f}\right)$ and for every $x$ such that $x \in Z$ holds $f(x)=x$. Then

(i) $\operatorname{cosec} \cdot \frac{1}{f}$ is differentiable on $Z$, and

(ii) for every $x$ such that $x \in Z$ holds $\left(\operatorname{cosec} \cdot \frac{1}{f}\right)_{{ }^{\prime} Z}^{\prime}(x)=$ $\frac{\text { (the function } \cos )\left(\frac{1}{x}\right)}{x^{2} \cdot(\text { the function } \sin )\left(\frac{1}{x}\right)^{2}}$.

(10) Suppose $Z \subseteq \operatorname{dom}\left(\sec \cdot\left(f_{1}+c f_{2}\right)\right)$ and $f_{2}={ }_{\mathbb{Z}}^{2}$ and for every $x$ such that $x \in Z$ holds $f_{1}(x)=a+b \cdot x$. Then

(i) $\sec \cdot\left(f_{1}+c f_{2}\right)$ is differentiable on $Z$, and

(ii) for every $x$ such that $x \in Z$ holds $\left(\sec \cdot\left(f_{1}+c f_{2}\right)\right)_{\curlyvee Z}^{\prime}(x)=$ $\frac{(b+2 \cdot c \cdot x) \cdot(\text { the function } \sin )\left(a+b \cdot x+c \cdot x^{2}\right)}{(\text { the function } \cos )\left(a+b \cdot x+c \cdot x^{2}\right)^{2}}$.

(11) Suppose $Z \subseteq \operatorname{dom}\left(\operatorname{cosec} \cdot\left(f_{1}+c f_{2}\right)\right)$ and $f_{2}={ }_{\mathbb{Z}}^{2}$ and for every $x$ such that $x \in Z$ holds $f_{1}(x)=a+b \cdot x$. Then

(i) $\operatorname{cosec} \cdot\left(f_{1}+c f_{2}\right)$ is differentiable on $Z$, and

(ii) for every $x$ such that $x \in Z$ holds $\left(\operatorname{cosec} \cdot\left(f_{1}+c f_{2}\right)\right)_{{ }_{1 Z}}^{\prime}(x)=$ $-\frac{(b+2 \cdot c \cdot x) \cdot(\text { the function } \cos )\left(a+b \cdot x+c \cdot x^{2}\right)}{(\text { the function } \sin )\left(a+b \cdot x+c \cdot x^{2}\right)^{2}}$.

(12) Suppose $Z \subseteq \operatorname{dom}(\mathrm{sec} \cdot($ the function exp)). Then

(i) $\sec \cdot($ the function $\exp$ ) is differentiable on $Z$, and

(ii) for every $x$ such that $x \in Z$ holds (sec $\cdot($ the function $\exp ))_{{ }^{\prime}}^{\prime}(x)=$ $\frac{(\text { the function } \exp )(x) \cdot(\text { the function } \sin )((\text { the function } \exp )(x))}{\text { (the function } \cos )((\text { the function } \exp )(x))^{2}}$.

(13) Suppose $Z \subseteq \operatorname{dom}(\operatorname{cosec} \cdot($ the function exp)). Then

(i) $\operatorname{cosec} \cdot($ the function exp) is differentiable on $Z$, and 
(ii) for every $x$ such that $x \in Z$ holds $(\operatorname{cosec} \cdot(\text { the function } \exp ))^{\prime}{ }_{\mid Z}(x)=$ $-\frac{\text { (the function } \exp )(x) \cdot(\text { the function } \cos )((\text { the function } \exp )(x))}{(\text { the function sin) }(\text { (the function } \exp )(x))^{2}}$.

(14) Suppose $Z \subseteq \operatorname{dom}(\mathrm{sec} \cdot($ the function $\ln )$ ). Then

(i) $\sec \cdot($ the function $\ln$ ) is differentiable on $Z$, and

(ii) for every $x$ such that $x \in Z$ holds $\left(\sec \cdot(\right.$ the function $\ln ){ }_{{ }^{\prime} Z}^{\prime}(x)=$ $\frac{(\text { the function } \sin )((\text { the function } \ln )(x))}{x \cdot(\text { the function } \cos )((\text { the function } \ln )(x))^{2}}$.

(15) Suppose $Z \subseteq \operatorname{dom}(\operatorname{cosec} \cdot($ the function $\ln ))$. Then

(i) $\operatorname{cosec} \cdot($ the function $\ln )$ is differentiable on $Z$, and

(ii) for every $x$ such that $x \in Z$ holds $(\operatorname{cosec} \cdot(\text { the function } \ln ))^{\prime}{ }_{{ }_{Z}}(x)=$ $-\frac{(\text { the function } \cos )((\text { the function } \ln )(x))}{x \cdot(\text { the function } \sin )((\text { the function } \ln )(x))^{2}}$.

(16) Suppose $Z \subseteq \operatorname{dom}(($ the function $\exp ) \cdot \sec )$. Then

(i) (the function exp) - sec is differentiable on $Z$, and

(ii) for every $x$ such that $x \in Z$ holds ((the function $\exp ) \cdot \sec )^{\prime}{ }^{\prime}(x)=$ $\frac{\text { (the function } \exp )((\sec )(x)) \cdot(\text { the function } \sin )(x)}{(\text { the function } \cos )(x)^{2}}$.

(17) Suppose $Z \subseteq \operatorname{dom}(($ the function $\exp ) \cdot \operatorname{cosec})$. Then

(i) (the function exp) $\cdot \operatorname{cosec}$ is differentiable on $Z$, and

(ii) for every $x$ such that $x \in Z$ holds $((\text { the function } \exp ) \cdot \operatorname{cosec})^{\prime}{ }_{\mid Z}(x)=$ $-\frac{\text { (the function } \exp )((\operatorname{cosec})(x)) \cdot(\text { the function } \cos )(x)}{(\text { the function } \sin )(x)^{2}}$.

(18) Suppose $Z \subseteq \operatorname{dom}(($ the function $\ln ) \cdot \sec )$. Then

(i) (the function $\ln ) \cdot \sec$ is differentiable on $Z$, and

(ii) for every $x$ such that $x \in Z$ holds ((the function $\ln ) \cdot \sec )^{\prime}{ }_{Z Z}(x)=$ (the function $\sin )(x)$. (the function $\cos )(x)$.

(19) Suppose $Z \subseteq \operatorname{dom}(($ the function $\ln ) \cdot \operatorname{cosec})$. Then

(i) (the function $\ln ) \cdot \operatorname{cosec}$ is differentiable on $Z$, and

(ii) for every $x$ such that $x \in Z$ holds ((the function $\ln ) \cdot \operatorname{cosec})_{{ }^{\prime}}^{\prime}(x)=$ $-\frac{(\text { the function } \cos )(x)}{\text { (the function } \sin )(x)}$.

(20) Suppose $Z \subseteq \operatorname{dom}\left(\left(\begin{array}{l}n \\ \mathbb{Z}\end{array}\right) \cdot \sec \right)$ and $1 \leq n$. Then

(i) $\quad\left(\begin{array}{l}n \\ \mathbb{Z}\end{array}\right) \cdot$ sec is differentiable on $Z$, and

(ii) for every $x$ such that $x \in Z$ holds $((\underset{\mathbb{Z}}{n}) \cdot \sec )^{\prime}{ }_{\mid Z}^{\prime}(x)=\frac{n \cdot(\text { the function } \sin )(x)}{(\text { the function } \cos )(x)_{\mathbb{Z}}^{n+1}}$.

(21) Suppose $Z \subseteq \operatorname{dom}\left(\left(\begin{array}{l}n \\ \mathbb{Z}\end{array}\right) \cdot \operatorname{cosec}\right)$ and $1 \leq n$. Then

(i) $\quad\left(\begin{array}{l}n \\ \mathbb{Z}\end{array}\right) \cdot \operatorname{cosec}$ is differentiable on $Z$, and

(ii) for every $x$ such that $x \in Z$ holds $((\underset{\mathbb{Z}}{n}) \cdot \operatorname{cosec})_{{ }_{i Z}}^{\prime}(x)=$ $-\frac{n \cdot(\text { the function } \cos )(x)}{(\text { the function } \sin )(x)_{\mathbb{Z}}^{n+1}}$.

(22) Suppose $Z \subseteq \operatorname{dom}\left(\sec -\mathrm{id}_{Z}\right)$. Then

(i) $\quad \mathrm{sec}-\mathrm{id}_{Z}$ is differentiable on $Z$, and

(ii) for every $x$ such that $x \in Z$ holds $\left(\sec -\operatorname{id}_{Z}\right)_{\ulcorner Z}^{\prime}(x)=$ $\frac{\text { (the function } \sin )(x)-(\text { the function } \cos )(x)^{2}}{(\text { the function } \cos )(x)^{2}}$. 
(23) Suppose $Z \subseteq \operatorname{dom}\left(-\operatorname{cosec}-\mathrm{id}_{Z}\right)$. Then

(i) $-\operatorname{cosec}-\mathrm{id}_{Z}$ is differentiable on $Z$, and

(ii) for every $x$ such that $x \in Z$ holds $\left(-\operatorname{cosec}-\operatorname{id}_{Z}\right)_{{ }^{\prime}}^{\prime}(x)=$ $\frac{(\text { the function } \cos )(x)-(\text { the function } \sin )(x)^{2}}{(\text { the function } \sin )(x)^{2}}$.

(24) Suppose $Z \subseteq \operatorname{dom}(($ the function $\exp )$ sec). Then

(i) (the function exp) sec is differentiable on $Z$, and

(ii) for every $x$ such that $x \in Z$ holds ((the function exp) $\sec )_{{ }_{Z}}^{\prime}(x)=$ $\frac{(\text { the function } \exp )(x)}{(\text { the function } \cos )(x)}+\frac{\text { (the function } \exp )(x) \cdot(\text { the function } \sin )(x)}{(\text { the function } \cos )(x)^{2}}$.

(25) Suppose $Z \subseteq \operatorname{dom}(($ the function $\exp ) \operatorname{cosec})$. Then

(i) (the function exp) cosec is differentiable on $Z$, and

(ii) for every $x$ such that $x \in Z$ holds ((the function exp) $\operatorname{cosec}^{\prime}{ }^{\prime}{ }_{Z}(x)=$ $\frac{(\text { the function } \exp )(x)}{\text { (the function } \sin )(x)}-\frac{(\text { the function } \exp )(x) \cdot(\text { the function } \cos )(x)}{(\text { the function } \sin )(x)^{2}}$.

(26) Suppose $Z \subseteq \operatorname{dom}\left(\frac{1}{a}(\sec \cdot f)-\operatorname{id}_{Z}\right)$ and for every $x$ such that $x \in Z$ holds $f(x)=a \cdot x$ and $a \neq 0$. Then

(i) $\frac{1}{a}(\mathrm{sec} \cdot f)-\mathrm{id}_{Z}$ is differentiable on $Z$, and

(ii) for every $x$ such that $x \in Z$ holds $\left(\frac{1}{a}(\sec \cdot f)-\operatorname{id}_{Z}\right)^{\prime}{ }_{\mid Z}(x)=$ $\frac{\text { (the function } \sin )(a \cdot x)-(\text { the function } \cos )(a \cdot x)^{2}}{(\text { the function } \cos )(a \cdot x)^{2}}$.

(27) Suppose $Z \subseteq \operatorname{dom}\left(\left(-\frac{1}{a}\right)(\operatorname{cosec} \cdot f)-\mathrm{id}_{Z}\right)$ and for every $x$ such that $x \in Z$ holds $f(x)=a \cdot x$ and $a \neq 0$. Then

(i) $\left(-\frac{1}{a}\right)(\operatorname{cosec} \cdot f)-\mathrm{id}_{Z}$ is differentiable on $Z$, and

(ii) for every $x$ such that $x \in Z$ holds $\left(\left(-\frac{1}{a}\right)(\operatorname{cosec} \cdot f)-\operatorname{id}_{Z}\right)^{\prime}{ }_{\mid Z}(x)=$ $\frac{(\text { the function } \cos )(a \cdot x)-(\text { the function } \sin )(a \cdot x)^{2}}{(\text { the function } \sin )(a \cdot x)^{2}}$.

(28) Suppose $Z \subseteq \operatorname{dom}(f$ sec $)$ and for every $x$ such that $x \in Z$ holds $f(x)=$ $a \cdot x+b$. Then

(i) $f$ sec is differentiable on $Z$, and

(ii) for every $x$ such that $x \in Z$ holds $(f \text { sec })^{\prime}{ }_{Z}(x)=\frac{a}{\text { (the function } \cos )(x)}+$ $\frac{(a \cdot x+b) \cdot(\text { the function } \sin )(x)}{(\text { the function } \cos )(x)^{2}}$.

(29) Suppose $Z \subseteq \operatorname{dom}(f \operatorname{cosec})$ and for every $x$ such that $x \in Z$ holds $f(x)=a \cdot x+b$. Then

(i) $f$ cosec is differentiable on $Z$, and

(ii) for every $x$ such that $x \in Z$ holds $(f \operatorname{cosec})^{\prime}{ }_{\mid Z}(x)=\frac{a}{\text { (the function } \sin )(x)}-$ $\frac{(a \cdot x+b) \cdot(\text { the function } \cos )(x)}{(\text { the function } \sin )(x)^{2}}$.

(30) Suppose $Z \subseteq \operatorname{dom}(($ the function $\ln )$ sec). Then

(i) (the function $\ln$ ) sec is differentiable on $Z$, and

(ii) for every $x$ such that $x \in Z$ holds ((the function $\ln ) \sec ^{\prime}{ }_{{ }^{\prime}}(x)=$ $\frac{\frac{1}{\text { (the function } \cos )(x)}}{x}+\frac{(\text { the function } \ln )(x) \cdot(\text { the function } \sin )(x)}{(\text { the function } \cos )(x)^{2}}$.

(31) Suppose $Z \subseteq \operatorname{dom}(($ the function $\ln )$ cosec $)$. Then 
(i) (the function $\ln$ ) cosec is differentiable on $Z$, and

(ii) for every $x$ such that $x \in Z$ holds ((the function $\ln ) \operatorname{cosec}^{\prime}{ }_{{ }^{\prime}}(x)=$ $\frac{\frac{1}{(\text { the function } \sin )(x)}}{x}-\frac{\text { (the function } \ln )(x) \cdot(\text { the function } \cos )(x)}{(\text { the function } \sin )(x)^{2}}$.

(32) Suppose $Z \subseteq \operatorname{dom}\left(\frac{1}{f}\right.$ sec $)$ and for every $x$ such that $x \in Z$ holds $f(x)=x$. Then

(i) $\frac{1}{f}$ sec is differentiable on $Z$, and

(ii) for every $x$ such that $x \in Z$ holds $\left(\frac{1}{f} \sec \right)^{\prime}{ }_{\mid Z}(x)=-\frac{\frac{1}{(\text { the function } \cos )(x)}}{x^{2}}+$ $\frac{\frac{(\text { the function } \sin )(x)}{x}}{\text { (the function } \cos )(x)^{2}}$.

(33) Suppose $Z \subseteq \operatorname{dom}\left(\frac{1}{f} \operatorname{cosec}\right)$ and for every $x$ such that $x \in Z$ holds $f(x)=x$. Then

(i) $\frac{1}{f}$ cosec is differentiable on $Z$, and

(ii) for every $x$ such that $x \in Z$ holds $\left(\frac{1}{f} \operatorname{cosec}\right)^{\prime}{ }_{\mid Z}^{\prime}(x)=-\frac{\frac{1}{(\text { the function } \sin )(x)}}{x^{2}}-$

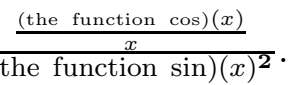

(34) Suppose $Z \subseteq \operatorname{dom}(\mathrm{sec} \cdot($ the function $\sin ))$. Then

(i) $\sec \cdot$ (the function $\sin$ ) is differentiable on $Z$, and

(ii) for every $x$ such that $x \in Z$ holds $(\mathrm{sec} \cdot(\text { the function } \sin ))_{{ }^{\prime} Z}^{\prime}(x)=$ $\frac{\text { (the function } \cos )(x) \cdot(\text { the function } \sin )((\text { the function } \sin )(x))}{(\text { the function } \cos )((\text { the function } \sin )(x))^{2}}$.

(35) Suppose $Z \subseteq \operatorname{dom}(\mathrm{sec} \cdot($ the function $\cos )$ ). Then

(i) $\sec \cdot($ the function cos) is differentiable on $Z$, and

(ii) for every $x$ such that $x \in Z$ holds (sec (the function $\cos ){ }^{\prime}{ }_{\mid Z}(x)=$ $-\frac{\text { (the function } \sin )(x) \cdot(\text { the function } \sin )((\text { the function } \cos )(x))}{(\text { the function } \cos )(\text { (the function } \cos )(x))^{2}}$.

(36) Suppose $Z \subseteq \operatorname{dom}(\operatorname{cosec} \cdot($ the function $\sin ))$. Then

(i) $\operatorname{cosec} \cdot($ the function sin) is differentiable on $Z$, and

(ii) for every $x$ such that $x \in Z$ holds $(\operatorname{cosec} \cdot(\text { the function } \sin ))_{{ }^{\prime}}^{\prime}(x)=$ $-\frac{(\text { the function } \cos )(x) \cdot(\text { the function } \cos )((\text { the function } \sin )(x))}{(\text { the function } \sin )((\text { the function } \sin )(x))^{2}}$.

(37) Suppose $Z \subseteq \operatorname{dom}(\operatorname{cosec} \cdot($ the function $\cos ))$. Then

(i) $\operatorname{cosec} \cdot($ the function $\cos$ ) is differentiable on $Z$, and

(ii) for every $x$ such that $x \in Z$ holds $(\operatorname{cosec} \cdot(\text { the function } \cos ))_{{ }^{\prime}}^{\prime}(x)=$ (the function $\sin )(x) \cdot($ the function $\cos )(($ the function $\cos )(x))$.

(38) Suppose $Z \subseteq \operatorname{dom}(\mathrm{sec} \cdot($ the function tan)). Then

(i) $\mathrm{sec} \cdot($ the function $\tan$ ) is differentiable on $Z$, and

(ii) for every $x$ such that $x \in Z$ holds (sec.(the function $\tan ))_{{ }^{\prime} Z}^{\prime}(x)=$ $\frac{(\text { the function } \sin )((\text { the function } \tan )(x))}{(\text { the function } \cos )(x)^{2}}$ $\frac{(\text { the function } \cos )(x)^{2}}{\text { (the function cos)((the function } \tan )(x))^{\mathbf{2}}}$.

(39) Suppose $Z \subseteq \operatorname{dom}(\mathrm{sec} \cdot($ the function cot)). Then

(i) $\mathrm{sec} \cdot($ the function cot) is differentiable on $Z$, and 
(ii) for every $x$ such that $x \in Z$ holds ( $\sec \cdot($ the function $\cot ))^{\prime}{ }^{\prime}(x)=$ $-\frac{\frac{\text { (the function } \sin )((\text { the function } \cot )(x))}{(\text { the function } \sin )(x)^{2}}}{\text { (the function cos })((\text { the function } \cot )(x))^{2}}$.

(40) Suppose $Z \subseteq \operatorname{dom}(\operatorname{cosec} \cdot($ the function $\tan ))$. Then

(i) $\operatorname{cosec} \cdot($ the function $\tan )$ is differentiable on $Z$, and

(ii) for every $x$ such that $x \in Z$ holds $(\operatorname{cosec} \cdot(\text { the function } \tan ))_{{ }^{\prime}}^{\prime}(x)=$ $-\frac{\frac{\text { (the function } \cos )((\text { the function } \tan )(x))}{(\text { the function } \cos )(x)^{2}}}{\text { (the function sin })((\text { the function } \tan )(x))^{2}}$.

(41) Suppose $Z \subseteq \operatorname{dom}(\operatorname{cosec} \cdot($ the function $\cot ))$. Then

(i) $\operatorname{cosec} \cdot($ the function cot) is differentiable on $Z$, and

(ii) for every $x$ such that $x \in Z$ holds $(\operatorname{cosec} \cdot(\text { the function } \cot ))_{{ }^{\prime}}^{\prime}(x)=$ $\frac{\text { (the function } \cos )((\text { the function } \cot )(x))}{(\text { the function } \sin )(x)^{2}}$

$\frac{(\text { the function } \sin )(x)^{2}}{\text { (the function } \sin )((\text { the function } \cot )(x))^{2}}$.

(42) Suppose $Z \subseteq \operatorname{dom}(($ the function $\tan )$ sec). Then

(i) (the function tan) sec is differentiable on $Z$, and

(ii) for every $x$ such that $x \in Z$ holds ((the function $\tan ) \sec ^{\prime}{ }_{\mid Z}(x)=$ $\frac{\frac{1}{(\text { the function } \cos )(x)^{2}}}{\text { (the function } \cos )(x)}+\frac{(\text { the function } \tan )(x) \cdot(\text { the function } \sin )(x)}{(\text { the function } \cos )(x)^{2}}$.

(43) Suppose $Z \subseteq \operatorname{dom}$ ((the function cot) sec). Then

(i) (the function cot) sec is differentiable on $Z$, and

(ii) for every $x$ such that $x \in Z$ holds ((the function cot) $\sec )^{\prime}{ }_{Z}(x)=$ $-\frac{\frac{1}{(\text { the function } \sin )(x)^{2}}}{(\text { the function } \cos )(x)}+\frac{(\text { the function } \cot )(x) \cdot(\text { the function } \sin )(x)}{(\text { the function } \cos )(x)^{2}}$.

(44) Suppose $Z \subseteq \operatorname{dom}(($ the function tan) cosec). Then

(i) (the function tan) cosec is differentiable on $Z$, and

(ii) for every $x$ such that $x \in Z$ holds ((the function $\tan ) \operatorname{cosec}^{\prime}{ }_{Y Z}(x)=$ $\frac{\frac{1}{(\text { the function } \cos )(x)^{2}}}{\text { (the function } \sin )(x)}-\frac{(\text { the function } \tan )(x) \cdot(\text { the function } \cos )(x)}{\text { (the function } \sin )(x)^{2}}$.

(45) Suppose $Z \subseteq \operatorname{dom}(($ the function cot) $\operatorname{cosec})$. Then

(i) (the function cot) cosec is differentiable on $Z$, and

(ii) for every $x$ such that $x \in Z$ holds ((the function cot) $\operatorname{cosec})^{\prime}{ }_{Z}(x)=$ $-\frac{\frac{1}{(\text { the function } \sin )(x)^{2}}}{(\text { the function } \sin )(x)}-\frac{(\text { the function } \cot )(x) \cdot(\text { the function } \cos )(x)}{(\text { the function } \sin )(x)^{2}}$.

\section{REFERENCES}

[1] Grzegorz Bancerek. The ordinal numbers. Formalized Mathematics, 1(1):91-96, 1990.

[2] Czesław Byliński. Partial functions. Formalized Mathematics, 1(2):357-367, 1990.

[3] Krzysztof Hryniewiecki. Basic properties of real numbers. Formalized Mathematics, $1(\mathbf{1}): 35-40,1990$.

[4] Jarosław Kotowicz. Partial functions from a domain to a domain. Formalized Mathematics, 1(4):697-702, 1990.

[5] Jarosław Kotowicz. Partial functions from a domain to the set of real numbers. Formalized Mathematics, 1(4):703-709, 1990.

[6] Jarosław Kotowicz. Real sequences and basic operations on them. Formalized Mathematics, 1(2):269-272, 1990. 
[7] Rafał Kwiatek. Factorial and Newton coefficients. Formalized Mathematics, 1(5):887-890, 1990.

[8] Konrad Raczkowski. Integer and rational exponents. Formalized Mathematics, 2(1):125130, 1991.

[9] Konrad Raczkowski and Paweł Sadowski. Real function differentiability. Formalized Mathematics, 1(4):797-801, 1990.

[10] Konrad Raczkowski and Paweł Sadowski. Topological properties of subsets in real numbers. Formalized Mathematics, 1(4):777-780, 1990.

[11] Yasunari Shidama. The Taylor expansions. Formalized Mathematics, 12(2):195-200, 2004.

[12] Andrzej Trybulec. Subsets of complex numbers. To appear in Formalized Mathematics.

[13] Andrzej Trybulec. Tarski Grothendieck set theory. Formalized Mathematics, 1(1):9-11, 1990.

[14] Andrzej Trybulec and Czesław Byliński. Some properties of real numbers. Formalized Mathematics, 1(3):445-449, 1990.

[15] Zinaida Trybulec. Properties of subsets. Formalized Mathematics, 1(1):67-71, 1990.

[16] Edmund Woronowicz. Relations defined on sets. Formalized Mathematics, 1(1):181-186, 1990.

[17] Yuguang Yang and Yasunari Shidama. Trigonometric functions and existence of circle ratio. Formalized Mathematics, 7(2):255-263, 1998.

Received July 9, 2007 